\title{
Evapotranspiração e coeficiente de cultivo do algodoeiro BRS-200 Marrom, irrigado
}

\author{
José R. C. Bezerra ${ }^{1}$, Pedro V. de Azevedo ${ }^{2}$, Bernardo B. da Silva² \& José M. Dias ${ }^{3}$
}

\begin{abstract}
RESU MO
Experimento conduzido na Embrapa, em Barbalha, CE, nos anos de 2003 e 2005, objetivou a estimativa da evapotranspiração da cultura e do coeficiente de cultivo do algodoeiro BRS-200 Marrom. Sensores de radiação solar global; saldo de radiação; temperatura do ar (bulbos seco e úmido) e velocidade do vento nos níveis de 0,30 e 1,50 m acima da copa da cultura e fluxo de calor no solo foram instalados e os dados coletados por um sistema automático de aquisição de dados. A evapotranspiração da cultura ( $\left.E T_{C}\right)$ e a evapotranspiração de referência $\left(E T_{0}\right)$ foram estimadas pelos métodos da razão de Bowen e Penmam-Monteith, respectivamente, enquanto o coeficiente de cultivo $(\mathrm{KC})$ foi determinado pela razão $E T_{C} / E T_{0}$. A ETc da cultura variou em função de sua fenologia, obtendo-se valores médios de $3,8 \mathrm{~mm} \mathrm{~d}^{-1}$ no período da emergência a $10 \%$ da cobertura de solo (Fase I); $5,0 \mathrm{~mm} \mathrm{~d}^{-1}$ no período do crescimento vegetativo (Fase II); 5,9 $\mathrm{mm} \mathrm{d}^{-1}$ no período do desenvolvimento reprodutivo (Fase III) e $5,4 \mathrm{~mm} \mathrm{~d}^{-1}$ no período de maturação (Fase IV). $0 \mathrm{Kc}$ pode ser definido em função dos dias após a emergência, pela equação $K c=-0,00006 D^{2} E^{2}+0,009 D A E+0,632$.
\end{abstract}

Palavras-chave: Gossypium hirsutum L., irrigação, consumo hídrico, balanço de energia

\section{Evapotranspiration and crop coefficient of irrigated cotton crop, cultivar BRS-200 Marrom}

\begin{abstract}
Field experiments were carried out at the EMBRAPA, Barbalha, CE, during the growing seasons of 2003 and 2005 with the objective of estimating the evapotranspiration and crop coefficient of irrigated BRS-200 Marrom cotton cultivar. For this sensors of solar radiation, net radiation, dry and wet bulbs air temperature and wind speed at the 0.30 and $1.50 \mathrm{~m}$ levels above crop canopy and soil heat flux were installed and data were collected by a data acquisition system. Crop $\left(E T_{c}\right)$ and reference $\left(E_{0}\right)$ evapotranspiration were estimated by the Bowen ratio and Penman-Monteith methods, respectively, while the crop coefficient $\left(\mathrm{K}_{c}\right)$ was estimated by the ratio of $E T_{c}$ to $E T_{0}$. The $E T_{c}$ changed throughout the phonological cycle of the cotton crop, with mean values of $3.8 \mathrm{~mm} \mathrm{~d}^{-1}$ in the period from emergence to $10 \%$ of soil cover (phase I), $5.0 \mathrm{~mm} \mathrm{~d}^{-1}$ in the period of vegetative growth (phase II), $5.9 \mathrm{~mm} \mathrm{~d}^{-1}$ in the period of reproductive development (phase III) and $5.4 \mathrm{~mm} \mathrm{~d}^{-1}$ in the maturation period (phase IV). The $\mathrm{K}_{\mathrm{c}}$ may be estimated as a function of the days after emergence (DAE) as: $K_{c}=-0.00006 D_{A E}^{2}+0.009 D A E+0.632$.
\end{abstract}

Key words: Gossypium hirsutum L., irrigation, crop water use, energy balance

\footnotetext{
1 Pesquisador da Embrapa Algodão, Rua 0 svaldo Cruz, 1143, Centenário, CEP 58428-095, Campina Grande, PB. Fone: (83) 3182-4300, (83) $3182-4367$. E-mail: renato@cnpa.embrapa.br

2 UACA/UFCG, Av. Aprígio Veloso, 882, Bodocongó, CEP 58429-140, Campina Grande, PB. Fone: (83) 3310-1199. E-mail: pvieira@dca.ufcg.edu.br; bernardo@dca.ufcg.edu.br

${ }^{3}$ Assistente de Pesquisa da Embrapa Algodão. E-mail: marcelo@cnpa.embrapa.br
} 


\section{INTRODUÇÃO}

As condições edafoclimáticas do semiárido brasileiro são favoráveis à cotonicultura, visto que suportam as irregularidades na distribuição da precipitação pluvial; trata-se de uma atividade agrícola de suma importância socioeconômica para a região, pois agrega um grande contingente de mão-de-obra no campo e na cidade, além de impulsionar o parque têxtil do País, o qual necessita de matéria-prima para atender à sua demanda; entretanto, apesar dessa cultura já ter ocupado uma área bastante significativa na região, chegando a mais de 3.000.000 ha (Beltrão et al., 2008), o estabelecimento da praga do bicudo aliado a problemas de ordem conjuntural, provocou uma redução bastante acentuada da área cultivada.

Diante desta conjuntura e visando obter novas alternativas para a agricultura da região, a Embrapa Algodão desenvolveu uma cultivar de fibra colorida a partir do algodoeiro arbóreo com fibra colorida, ou seja, a BRS-200 Marrom, que é um "bulk" constituído pela mistura, em partes iguais, de três linhagens derivadas de algodoeiro arbóreo, com ciclo produtivo de três anos; apesar de ter sido desenvolvida para o regime de sequeiro devido às suas características de tolerância ao déficit hídrico, pode ser cultivada em regime de irrigação, com alto potencial produtivo. $\mathrm{O}$ cultivo deste material poderá possibilitar melhorias no processo produtivo da agricultura da região, por se tratar de um material rústico, de fibra naturalmente colorida, oferecendo vantagens econômicas ao cotonicultor em virtude do melhor preço da fibra no mercado e por ter maior produtividade que o algodoeiro arbóreo cultivado em regime de sequeiro. Em decorrência de sua coloração natural, o tingimento não é necessário no processamento industrial, acarretando dois benefícios diretos, em que o primeiro diz respeito ao meio ambiente, uma vez que no tingimento artificial se utiliza um grande volume de água produzindo resíduos altamente poluentes e o segundo se refere ao enorme potencial de mercado dos países do primeiro mundo, cuja demanda por produtos de origem natural, principalmente na Europa e no Japão, vem aumentando de forma considerável, o que torna seu potencial de expansão elevado.

Considerando-se esses aspectos, é indispensável um ajuste tecnológico no processo produtivo desse tipo de algodão, para que o produtor nordestino possa usufruir dos benefícios potenciais. Neste contexto, a irrigação poderá permitir a obtenção de maiores produtividades com a oferta de água às plantas no momento oportuno, possibilitando o seu máximo potencial produtivo; diminuição dos riscos decorrentes da má distribuição espacial e temporal das chuvas e a possibilidade da obtenção de uma fibra de melhor qualidade, em razão da intensidade de brilho solar, característica da região no período em que se pratica a agricultura irrigada; todavia, para a obtenção de altos rendimentos e maior eficiência no uso de água é necessário se conhecer as necessidades hídricas da cultura de modo que se possa oferecer às plantas, a quantidade de água adequada para os seus processos metabólicos e fisiológicos (Azevedo et al., 1993; Azevedo et al., 2003). Por sua vez, Silva \& Rao (2005), afirmam que a retomada da produção no Nordeste do Brasil está condicionada à aplicação de novas tecnologias, principalmente às relacionadas ao uso eficiente da água que provocará benefícios ambientais e redução nos custos de produção.

Como nas regiões áridas e semiáridas a disponibilidade de água no solo condiciona significativamente a produção agrícola, a utilização da irrigação tem assegurado o crescimento e o desenvolvimento das culturas aumentando substancialmente a produtividade; todavia e se considerando que a irrigação é a principal atividade consumidora de água, com consequente aumento dos custos com energia, além de concorrer pelos recursos hídricos e energéticos com os setores industrial e urbano, torna-se oportuna a realização de estudos que definam o momento de se efetuar as irrigações e a quantidade de água que deverá ser aplicada, visando atender às necessidades hídricas das plantas. $\mathrm{O}$ manejo eficiente da irrigação possibilita a economia desses recursos, a otimização do uso dos insumos agrícolas e a obtenção de maiores retornos econômicos, o que viabilizará o aumento da área irrigada no País (Amorim Neto, 1995). Para efetivação de um manejo eficiente, o conhecimento das necessidades hídricas da cultura é essencial e o método do balanço de energia baseado na razão de Bowen tem sido bastante utilizado em função de sua relativa simplicidade e precisão nos resultados (Gavilán \& Berengena, 2007; Silberstein et al., 2003; Silva et al., 2007; Teixeira et al., 2007; Zhang et al., 2008). Este método se baseia no princípio da conservação de energia onde o balanço dos fluxos de entrada e saída de energia no volume de controle, representado pela área vegetada, permite a determinação da energia utilizada pela cultura no processo de transferência de água sob a forma de vapor para a atmosfera (Cardoso et al., 2005). Para Silberstein et al. (2003), os fluxos de energia que ocorrem em uma superfície vegetada são o saldo de radiação $(\mathrm{Rn})$, fluxo de calor latente (LE), fluxo de calor sensível (H), fluxo de calor no solo (G), energia armazenada na copa das árvores (S) e energia utilizada no processo fotossintético $(\mathrm{P})$, sendo os dois últimos negligenciados no balanço de energia devido a baixa representatividade em relação ao saldo de radiação e sua difícil contabilização. A razão de Bowen é inferida pelas medições das diferenças médias de temperatura e umidade através da distância vertical fixada acima de uma superfície homogênea (Cardoso et al., 2005; Souza, 2003).

Face ao exposto, objetivou-se com o presente estudo, a determinação da evapotranspiração e do coeficiente de cultivo do algodoeiro, cultivar BRS-200 Marrom, em seu primeiro ano de cultivo.

\section{MATERIAL E MÉTODOS}

O experimento de campo foi conduzido na área experimental da Embrapa, localizada no município de Barbalha, CE, com coordenadas geográficas: Latitude: $07^{\circ} 19^{\prime} \mathrm{S}$, Longitude: $39^{\circ} 18^{\prime} \mathrm{W}$ e Altitude: $415,74 \mathrm{~m}$, nos períodos de 29 de julho a 10 de novembro e de 02 de setembro a 15 de dezembro, para os anos de 2003 e 2005, respectivamente. A classificação climática de Barbalha, $\mathrm{CE}$, é do tipo $\mathrm{C}_{1} \mathrm{~S}_{2} \mathrm{~A}^{\prime} \mathrm{a}$ ' (Thorthwaite \& Mather, 1955). Trata-se de um clima seco subúmido, com grande excesso hídrico no inverno, megatérmico e com 
Tabela 1. Valores médios mensais das normais climatológicas, pressão atmosférica $(\mathrm{hPa})$, temperatura média $\left({ }^{\circ} \mathrm{C}\right)$, temperatura máxima média $\left({ }^{\circ} \mathrm{C}\right)$, temperatura mínima média $\left({ }^{\circ} \mathrm{C}\right)$, precipitação média $(\mathrm{mm})$, evaporação média $(\mathrm{mm})$; umidade relativa média $(\%)$, insolação total $(\mathrm{h})$ e nebulosidade do Município de Barbalha, CE, 1961-1990

\begin{tabular}{|c|c|c|c|c|c|c|c|c|c|}
\hline \multirow{2}{*}{ Mês } & \multicolumn{9}{|c|}{ Variáveis meteorológicas } \\
\hline & Pres & Tmed & Tmax & Tmin & Prec & Evap & UR & Ins & Neb \\
\hline Jan & 963,8 & 25,5 & 32,1 & 21,2 & 172,5 & 160,0 & 68,0 & 216,4 & 7,0 \\
\hline Fev & 964,3 & 24,8 & 30,9 & 21,1 & 191,4 & 124,3 & 74,0 & 191,9 & 7,0 \\
\hline Mar & 964,4 & 24,5 & 30,3 & 20,9 & 234,3 & 107,7 & 80,0 & 196,4 & 6,0 \\
\hline $\mathrm{Abr}$ & 964,9 & 24,5 & 30,1 & 21,2 & 209,8 & 100,5 & 79,0 & 206,1 & 6,0 \\
\hline Mai & 965,9 & 24,1 & 30,0 & 20,6 & 48,1 & 145,8 & 73,0 & 224,9 & 8,0 \\
\hline Jun & 967,4 & 23,8 & 28,4 & 19,3 & 20,8 & 161,3 & 67,0 & 240,0 & 5,0 \\
\hline Jul & 968,4 & 23,8 & 29,9 & 19,1 & 11,5 & 224,8 & 61,0 & 252,4 & 7,0 \\
\hline Ago & 967,3 & 24,9 & 31,7 & 18,1 & 5,6 & 268,7 & 53,0 & 281,8 & 3,0 \\
\hline Set & 966,2 & 26,2 & 33,3 & 20,1 & 5,2 & 292,8 & 49,0 & 276,4 & 4,0 \\
\hline Out & 963,8 & 26,7 & 34,1 & 21,1 & 2,5 & 262,9 & 51,0 & 268,9 & 5,0 \\
\hline Nov & 963,8 & 26,8 & 33,9 & 21,8 & 4,8 & 223,7 & 53,0 & 256,4 & 5,0 \\
\hline Dez & 963,8 & 26,3 & 33,1 & 21,7 & 92,2 & 216,1 & 55,0 & 234,4 & 6,0 \\
\hline Média & 965,4 & 25,2 & 31,5 & 20,5 & - & - & 63,6 & - & 5,8 \\
\hline Soma & & & & & $1.001,4$ & $2.288,6$ & & $2.848,0$ & \\
\hline
\end{tabular}

vegetação durante todo o ano. De acordo com os dados de Brasil (1992), as normais climatológicas para esse município no período de 1961-1990, são apresentadas na Tabela 1.

O trabalho foi realizado em um solo de textura argilosa, cuja caracterização físico hídrica encontra-se na Tabela 2 e a análise de fertilidade apresentou o seguinte resultado: $\mathrm{pH} 7,3$; $\mathrm{Ca}^{+2}+\mathrm{Mg}^{+2}=186 \mathrm{mmol}_{\mathrm{c}} \mathrm{dm}^{-3} ; \mathrm{Na}^{+}=4,9 \mathrm{mmol}_{\mathrm{c}} \mathrm{dm}^{-3} ; \mathrm{K}^{+}=$ $6,0 \mathrm{mmol}_{\mathrm{c}} \mathrm{dm}^{-3} ; \underline{\mathrm{S}}=196,9 \mathrm{mmol}_{\mathrm{c}} \mathrm{dm}^{-3} ; \mathrm{Al}=0,0 \mathrm{mmol}_{\mathrm{c}} \mathrm{dm}^{-3}$; $\mathrm{P}=12,1 \mathrm{mg} \mathrm{dm}^{-3}$ e matéria orgânica $=19,1 \mathrm{~g} \mathrm{~kg}^{-1}$.

A cultura estudada foi o algodão, cultivar BRS-200 Marrom, plantada em 23/07/2003 e 26/08/2005, respectivamente, em fileiras duplas em uma área de aproximadamente 1,0 ha, em um espaçamento de $1,00 \times 0,40 \mathrm{~m}$, com 10 a 12 plantas por metro.

O preparo do solo constou de uma aração com arado escarificador, seguido de duas gradagens cruzadas utilizandose grade niveladora. A adubação de fundação foi efetuada no fundo do sulco de plantio, na dose de $30,60 \mathrm{e} 10 \mathrm{~kg} \mathrm{ha}^{-1}, \mathrm{~N}$, $\mathrm{P}_{2} \mathrm{O}_{5}$ e $\mathrm{K}_{2} \mathrm{O}$, respectivamente, com o uso de sulfato de amônio, superfosfato triplo e cloreto de potássio enquanto a adubação de cobertura foi efetuada aos 30 e 45 dias após a emergência aplicando-se, em cada ocasião, $30 \mathrm{~kg} \mathrm{ha}^{-1}$ de $\mathrm{N}$ e $10 \mathrm{~kg} \mathrm{ha}^{-1}$ de $\mathrm{K}_{2} 0$, sob a forma de uréia e cloreto de potás-

Tabela 2. Análise textural e caracterização físico hídrica do solo da área experimental de Barbalha, CE

\begin{tabular}{lccccc}
\hline \multirow{2}{*}{ Granulometria $\left(\mathbf{g ~ k g}^{-1}\right)$} & \multicolumn{5}{c}{ Profundidade $(\mathbf{c m})$} \\
\cline { 2 - 6 } Areia & $\mathbf{0 - 1 0}$ & $\mathbf{1 1 - 2 0}$ & $\mathbf{2 1 - 3 0}$ & $\mathbf{3 0 - 4 0}$ & $\mathbf{4 0 - 6 0}$ \\
Silte & 398 & 378 & 337 & 317 & 297 \\
Argila & 189 & 189 & 189 & 169 & 169 \\
Classificação Textural & 413 & 433 & 474 & 514 & 534 \\
/Densidade do solo $\left(\mathrm{kg} \mathrm{dm}^{-3}\right)$ & Argiloso & Argiloso & Argiloso & Argiloso & Argiloso \\
/Densidade de partículas $\left(\mathrm{kg} \mathrm{dm}^{-3}\right)$ & 1,37 & 1,36 & 1,35 & 1,36 & \\
Porosidade $\left(\mathrm{m}^{3} \mathrm{~m}^{-3}\right)$ & 0,65 & 2,65 & 2,65 & 2,65 & 2,65 \\
Capacidade de campo $\left(\mathrm{m}^{3} \mathrm{~m}^{-3}\right)$ & 0,32 & 0,49 & 0,49 & 0,49 & 0,48 \\
Ponto de murcha $\left(\mathrm{m}^{3} \mathrm{~m}^{-3}\right)$ & 0,14 & 0,14 & 0,33 & 0,33 & 0,34 \\
Capacid. total de água no solo $(\mathrm{mm})$ & 23,5 & 27,6 & 26,8 & 0,15 & 0,15 \\
\hline
\end{tabular}

sio, respectivamente. O controle de plantas daninhas foi feito com capinas manuais através de enxada, mantendo-se a lavoura livre de plantas daninhas durante os primeiros sessenta dias após a emergência. Para o controle de pragas utilizou-se o Manejo Integrado de Pragas recomendado pela Embrapa Algodão, que consistiu na amostragem das pragas a cada 5 dias a partir da emergência das plantas até o final do ciclo da cultura, efetuando-se o combate às pragas sempre que se alcançou o nível de controle.

Efetuaram-se as irrigações com aspersores Agropolo, com bocais de 3,2 x 5,4 mm, trabalhando-se com uma pressão de serviço de $2,5 \mathrm{~atm}$ e espaçamento de $18 \times 12 \mathrm{~m}$, aplicandose água até uma profundidade de $0,60 \mathrm{~m}$, correspondente ao perfil atingido pelo sistema radicular da cultura (Bezerra et al., 2008). O solo estudado apresentou disponibilidade total de água (DTA) de 149,8 mm e as irrigações de reposição foram efetuadas antes da depleção alcançar $60 \%$ da DTA, conforme resultados obtidos por Souza et al. (2002). Visando melhorar o desempenho do sistema de irrigação, antes do plantio, foi efetuada uma avaliação do sistema buscando identificar horários de menor velocidade de vento, com vistas a obtenção de uma maior eficiência na aplicação da água. A primeira irrigação, que antecedeu o plantio da cultura, foi efetuada de modo a levar o solo à "capacidade de campo"; após o plantio, na primeira fase do ciclo fenológico da cultura, a cada quatro dias, foram efetuadas irrigações de modo a repor a água que havia sido consumida. A partir do estabelecimento da cultura as irrigações foram efetuadas uma vez por semana fazendo-se a reposição da água em função do consumo semanal, com base no balanço de energia, segundo a razão de Bowen. Durante a execução dos experimentos, a precipitação ocorrida foi coletada em um pluviômetro instalado na estação meteorológica do INMET, situado dentro da área experimental da Embrapa.

A colheita foi manual, de forma fracionada, efetuando-se a primeira quando cerca de $60 \%$ dos capulhos estavam abertos e a segunda colheita após a completa abertura do restante dos capulhos. 
Para obtenção dos dados meteorológicos necessários ao trabalho, em uma torre micrometeorológica foram instalados: dois piranômetros para medição da radiação solar global $\left(\mathrm{R}_{\mathrm{s}}\right)$ e refletida pela cultura $\left(\mathrm{R}_{\mathrm{r}}\right)$; um saldo radiômetro para medição do saldo de radiação $\left(R_{n}\right)$; dois psicrômetros com termopares de cobre e "constantan", instalados em dois níveis, mantidos a 0,30 e 1,50 m acima da copa da cultura, variando ao longo dos ciclos de cultivo em função do desenvolvimento da cultura, com a finalidade de medir as temperaturas do ar em bulbo seco e úmido; dois anemômetros para medir a velocidade do vento, em dois níveis, na mesma altura dos medidores de temperatura; ademais, dois fluxímetros foram instalados a $0,02 \mathrm{~m}$ de profundidade (Borges et al., 2008; Rodrigues, 2003; Silva et al., 2007; Vourlitis et al., 2002), para medir o fluxo de calor no solo, sendo um entre duas fileiras e o outro dentro da fileira de plantas. Esses sensores foram conectados a um sistema automático de aquisição de dados (Datalogger CR 10X, da Campbell Scientific) para coleta e armazenamento dos sinais emitidos pelos sensores, programou-se o datalogger para efetuar leituras dos sinais analógicos e digitais a cada $5 \mathrm{~s}$ e extrair e armazenar as médias em intervalos de $20 \mathrm{~min}$.

Visando à eliminação ou redução do efeito da advecção, a torre foi instalada a aproximadamente $80 \mathrm{~m}$ a partir do limite da área cultivada com o algodão, obedecendo a direção dos ventos predominantes de modo que o fluxo ocorresse sobre o dossel da cultura e com uma relação superior a 20:1, já considerada satisfatória por Heilman et al. (1989). Por sua vez, Stannard et al. (2004), informam que o intervalo de relação entre altura do sensor e a bordadura de 30 a 200:1 não resulta em erros significativos.

Para determinação da evapotranspiração de referência utilizou-se a metodologia proposta por Penman-Monteith, que passou por algumas parametrizações, tais como: resistência estomática de $70 \mathrm{~s} \mathrm{~m}^{-1}$ e altura da grama fixada em 0,12 m, proposta pelo boletim FAO-56 (Allen et al., 2006); com isto, a equação para obtenção da evapotranspiração de referência foi expressa como:

$$
\text { ETo }=\frac{0,408 \Delta(\mathrm{Rn}-\mathrm{G})+\gamma\left(\frac{900}{\mathrm{~T}+273}\right) \mathrm{u}_{2}\left(\mathrm{e}_{\mathrm{s}}-\mathrm{e}_{\mathrm{a}}\right)}{\Delta+\gamma\left(1+0,34 \mathrm{u}_{2}\right)}
$$

em que ETo é a evapotranspiração de referencia $\left(\mathrm{mm} \mathrm{d}^{-1}\right)$; Rn é o saldo de radiação $\left(\mathrm{MJ} \mathrm{m}^{-2} \mathrm{~d}^{-1}\right)$; $\mathrm{G}$ é o fluxo de calor no solo $\left(\mathrm{MJ} \mathrm{m}^{-2} \mathrm{~d}^{-1}\right)$; $\mathrm{T}$ é a temperatura média diária do ar $\left({ }^{\circ} \mathrm{C}\right) ; \mathrm{u}_{2}$ é a velocidade do vento média diária a $2 \mathrm{~m}$ de altura $\left(\mathrm{m} \mathrm{s}^{-1}\right)$; $\mathrm{e}_{\mathrm{s}}$ é a pressão da saturação do vapor média diária $(\mathrm{kPa}) ; \mathrm{e}_{\mathrm{a}}$ é a pressão atual de vapor média diária $(\mathrm{kPa}) ; \Delta$ é a declividade da curva de pressão de vapor $\left(\mathrm{kPa}^{\circ} \mathrm{C}^{-1}\right)$; e $\gamma$ é a constante psicrométrica $\left(\mathrm{kPa}^{\circ} \mathrm{C}^{-1}\right)$.

$$
\Delta=\frac{4098\left[0,6108 \exp \left(\frac{17,27 \mathrm{~T}}{\mathrm{~T}+237,3}\right)\right]}{(\mathrm{T}+237,3)^{2}}
$$

A constante psicrométrica $(\gamma)$ foi considerado constante, $\gamma=0,0642 \mathrm{kPa}^{\circ} \mathrm{C}^{-1}$, pois ele é uma função da pressão atmosférica, que varia muito pouco ao longo do ano $(96,54 \mathrm{kPa})$, e também do calor latente de evaporação da água, que é fracamente afetado pela temperatura, sendo recomendado um valor médio igual a 2,45 $\mathrm{MJ} \mathrm{kg}^{-1}$.

A evapotranspiração da cultura $\left(\mathrm{ET}_{\mathrm{c}}, \mathrm{em} \mathrm{mm} \mathrm{d}^{-1}\right)$ foi estimada pelo método da Razão de Bowen, a partir da equação simplificada do balanço de energia (Perez et al., 2008):

$$
\mathrm{Rn}+\mathrm{LE}+\mathrm{H}+\mathrm{G}=0
$$

donde $\mathrm{Rn}$ é o saldo de radiação, LE é o fluxo de calor latente e $\mathrm{H}$ é o fluxo de calor sensível e $\mathrm{G}$ é o fluxo de calor no solo, respectivamente, todos em $\mathrm{W} \mathrm{m}^{-2}$. Neste processo, as densidades de fluxo que chegam à camada vegetativa são positivas enquanto as que saem são negativas.

Estimou-se a razão de Bowen de acordo com a expressão (Perez et al., 2008; Rosenberg et al., 1983):

$$
\beta=\frac{\mathrm{H}}{\mathrm{LE}}=\frac{\mathrm{P}_{0} \mathrm{C}_{\mathrm{P}}}{\mathrm{L} \varepsilon}=\left(\frac{\mathrm{K}_{\mathrm{h}}}{\mathrm{K}_{\mathrm{w}}}\right) \frac{\frac{\partial \mathrm{T}}{\partial \mathrm{z}}}{\frac{\partial \mathrm{e}}{\partial \mathrm{z}}}=\gamma\left(\frac{\mathrm{K}_{\mathrm{h}}}{\mathrm{K}_{\mathrm{w}}}\right) \frac{\Delta \mathrm{T}}{\Delta \mathrm{e}}
$$

Assumindo-se que os coeficientes de transferência turbulenta nos processos de difusão do calor sensível e vapor d'água são aproximadamente iguais, isto é $\mathrm{K}_{\mathrm{h}} \cong \mathrm{K}_{\mathrm{w}}$, (Verma et al., 1978) e considerando-se que $[(\partial \mathrm{T} / \partial \mathrm{z}) /(\partial \mathrm{e} / \partial \mathrm{z})] \cong \Delta \mathrm{T} / \Delta \mathrm{e}$, a razão de Bowen pode ser obtida como:

$$
\beta=\gamma \frac{\Delta \mathrm{T}}{\Delta \mathrm{e}}
$$

donde $\mathrm{K}_{\mathrm{h}}$ e $\mathrm{K}_{\mathrm{w}}$ são os coeficientes de difusão turbulenta de calor sensível e vapor d'água, respectivamente $\left(\mathrm{m}^{2} \mathrm{~s}^{-1}\right)$; L é o calor latente de evaporação da água $\left(\mathrm{MJ} \mathrm{kg}^{-1}\right) ; \mathrm{C}_{\mathrm{p}}$ é o calor específico do ar seco a pressão constante $\left(\mathrm{MJ} \mathrm{kg}^{-1}{ }^{\circ} \mathrm{C}^{-1}\right)$; $\mathrm{P}_{0}$ é a pressão atmosférica média local $(\mathrm{kPa}) ; \varepsilon$ é a razão entre as massas moleculares da água e do ar seco $(0,622)$; $\Delta \mathrm{T}=\mathrm{T}_{2}-\mathrm{T}_{1}$ e $\Delta \mathrm{e}=\mathrm{e}_{2}-\mathrm{e}_{1}$ são as diferenças de temperatura do ar e da pressão parcial do vapor d'água atmosférico, medidas em dois níveis acima da superfície vegetada $(0,3$ e $1,5 \mathrm{~m}), \Delta \mathrm{z}=\mathrm{z}_{2}-\mathrm{z}_{1}$ e $\gamma=\mathrm{C}_{\mathrm{p}} \mathrm{P}_{0} / \mathrm{L} \varepsilon$ é a constante psicrométrica $\left(\mathrm{kPa}{ }^{\circ} \mathrm{C}^{-1}\right)$.

Obteve-se o fluxo de calor latente (LE) pela expressão:

$$
L E=-\left(\frac{R n+G}{1+\beta}\right)
$$

Para a obtenção da evapotranspiração da cultura $\left(E_{c}\right)$ em $\mathrm{mm} \mathrm{d}^{-1}$, dividiu-se LE pelo calor latente de vaporização $(\mathrm{L})$, integrando-se os valores médios coletados no Datalogger para o período diurno em que $\mathrm{Rn}-\mathrm{G}>0$, conforme o já utilizado por Lopes et al. (2001); Prueger et al. (1997).

Determinou-se o coeficiente de cultura $(\mathrm{Kc})$ com base na relação, recomendada por Farahani et al. (2008) e Reis et al. (2009):

$$
\mathrm{K}_{\mathrm{c}}=\frac{\mathrm{ET}_{\mathrm{c}}}{\mathrm{ET}_{\mathrm{o}}}
$$




\section{RESULTADOS E DISCUSSÃO}

O ciclo fenológico do primeiro ano do algodoeiro, cultivar BRS-200 Marrom, foi dividido em quatro fases (Tabela 3), em função da ocorrência dos principais processos fisiológicos, de acordo com Doorenbos \& Pruitt (1979).

Tabela 3. Ciclo fenológico do algodoeiro, cultivar BRS-200 Marrom, em suas diversas fases de desenvolvimento, para os ciclos de produção de: 2003 (29/07 a 10/11/2003) e 2005 (02/09 a 15/12/2005), em seu primeiro ano de produção, no município de Barbalha, CE

\begin{tabular}{llcc}
\hline \multicolumn{1}{c}{ Fases de sesenvolvimento } & $\begin{array}{c}\text { Dias após } \\
\text { emergência } \\
\text { (DAE) }\end{array}$ & $\begin{array}{c}\text { Duração } \\
\text { (dias) }\end{array}$ \\
Fase I & Emergência a 10\% de cobertura de solo & $1-15$ & 15 \\
Fase II & $10 \%$ de cobertura de solo ao início da floração & $16-54$ & 39 \\
Fase III Início da floração ao início da maturação & $55-84$ & 31 \\
Fase IV Início ao final da maturação & $85-105$ & 20 \\
\hline \multicolumn{4}{c}{ TOTAL } \\
\hline
\end{tabular}

Verificou-se, a partir das observações de campo, que a Fase I (da emergência das plantas a $10 \%$ de cobertura de solo) teve duração de 15 dias; a Fase II, correspondente à fase do desenvolvimento vegetativo (de $10 \%$ de cobertura do solo ao início da floração) durou 39 dias; a Fase III, relativa à fase de desenvolvimento reprodutivo (do início da floração ao início da maturação), durou 31 dias, e a Fase IV, correspondente à fase final do ciclo da cultura (do início ao final da maturação) foi de 20 dias. O ciclo de desenvolvimento do algodoeiro, cultivar BRS-200 Marrom, em seu primeiro ano de cultivo foi completado aos 105 dias após a emergência, caracterizando uma cultivar de ciclo curto (Bezerra et al., 2008).

Tem-se, na Figura 1, o comportamento da evapotranspiração diária, ao longo dos ciclos de 2003 e 2005, do desenvolvimento do algodoeiro, cultivar BRS-200 Marrom irrigado.

Percebe-se, pelos dados da Figura 1, que a evapotranspiração da cultura foi bastante variável ao longo do seu ciclo fenológico, com valores mínimos de $3,2 \mathrm{~mm} \mathrm{~d}^{-1}$ e $2,9 \mathrm{~mm} \mathrm{~d}^{-1}$ no início da Fase I nos anos de 2003 e 2005, respectivamente e valores máximos de 7,1 $\mathrm{mm} \mathrm{d}^{-1}$ na Fase IV, em 2003 e $7,0 \mathrm{~mm} \mathrm{~d}^{-1}$ na fase II em 2005. Esta grande variação ao longo do ciclo também foi observada por Rodrigues (2003) no algodoeiro herbáceo cultivar BRS 201; este comportamento pode estar associado às variações da demanda atmosférica que influenciam o processo, caracterizada pela radiação solar global, efeito da velocidade do vento e da umidade relativa, conforme referido por Farahani et al. (2008) e Rosenberg et al. (1983) e que pode ser visualizado na Figura 2 (A, B e C). Segundo Souza (2003), variações na taxa de evapotranspiração diária durante a mesma fase fenológica podem ser decorrentes das condições meteorológicas locais e da duração da referida fase. Por sua vez Blanc et al. (2008), informaram que o aumento da $\mathrm{ET}_{\mathrm{c}}$, decorrente da elevação da demanda evaporativa da atmosfera, dentre outros fatores, é causado pelo aumento da radiação solar global.

Observa-se ainda que, em geral, a evapotranspiração da cul-

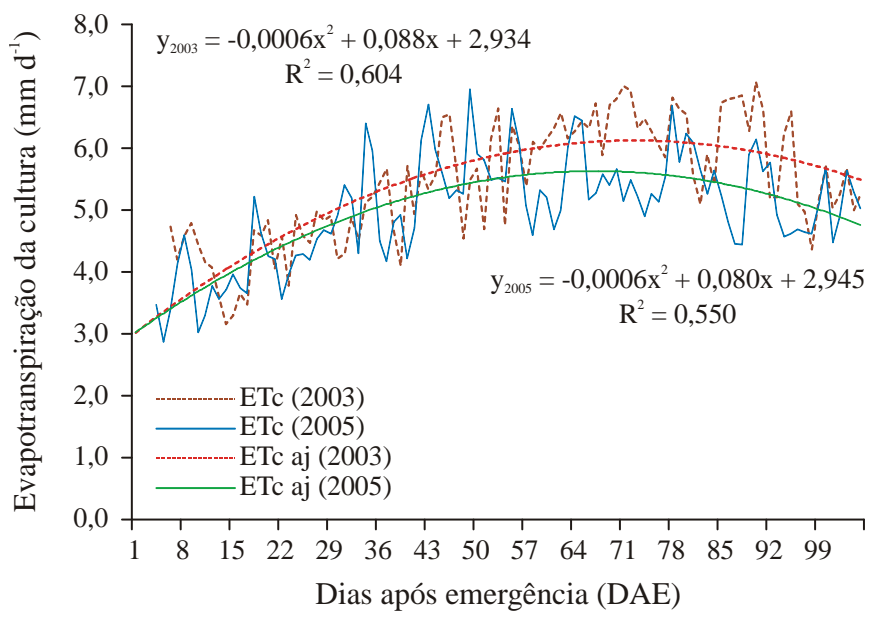

Figura 1. Variação da evapotranspiração diária do algodoeiro BRS 200Marrom irrigado, em Barbalha, CE, para os ciclos de desenvolvimento de 2003 e 2005

tura apresentou valores mais elevados após as irrigações ou chuva quando, em decorrência da maior disponibilidade de água no solo, houve maior taxa do processo de evapotranspiração (Rodrigues, 2003; Rosenberg et al., 1983), o que pode ser visualizado na Figura 2A e D. A exceção a esta situação ocorreu no final do ciclo do algodoeiro com dias consecutivos de intensa nebulosidade e precipitação (Figura 2D), acarretando queda na radiação solar global e velocidade do vento (Figura 2A e B) além de elevação na umidade relativa (Figura 2C), o que resultou em baixo valor da evapotranspiração da cultura, mesmo com elevado conteúdo de umidade do solo.

Quando se analisam os valores médios da evapotranspiração diária por fases do ciclo fenológico para os dois anos de observação (Tabela 4), nota-se um valor mínimo de $3,8 \mathrm{~mm} \mathrm{~d}^{-1}$ no início do desenvolvimento vegetativo (Fase I) e um máximo de $5,9 \mathrm{~mm} \mathrm{~d}^{-1}$ na fase de desenvolvimento reprodutivo (Fase III). Constata-se ainda nesta tabela, que a evapotranspiração da cultura foi crescente da Fase I à Fase III, quando alcançou o máximo, voltando em seguida a decrescer o que está de acordo com o observado por Bezerra et al. (1994), para a cultivar CNPA 6H; Azevedo et al. (1993) para a cultivar CNPA Precoce 1 e Pereira et al. (1997) para as cultivares CNPA Precoce 1 e CNPA 7H). Rodrigues (2003), trabalhando com a cultivar BRS 201, observou valores de evapotranspiração crescente da fase I para a II, havendo redução na fase III, alcançando o máximo na fase IV. Observando-se os dados obtidos por esse pesquisador, verifica-se que o critério adotado para estabelecer os períodos de cada fase fenológica foi diferente daqueles dos autores citados acima, razão pela qual se observa esta discrepância. Por sua vez, Bezerra et al. (2003), Jalota et al. (2006), Luz et al. (1997) e Radin et al. (1992), informam que o período do desenvolvimento reprodutivo do algodoeiro herbáceo, que corresponde à fase III, é o mais crítico ao déficit de umidade no solo. Por outro lado, analisando-se o desenvolvimento da cultura do algodão, pode-se observar que esta aumenta seu consumo em função do seu crescimento até alcançar o máximo na fase de desenvolvimento reprodutivo e a partir deste ponto, com o início da senescên- 


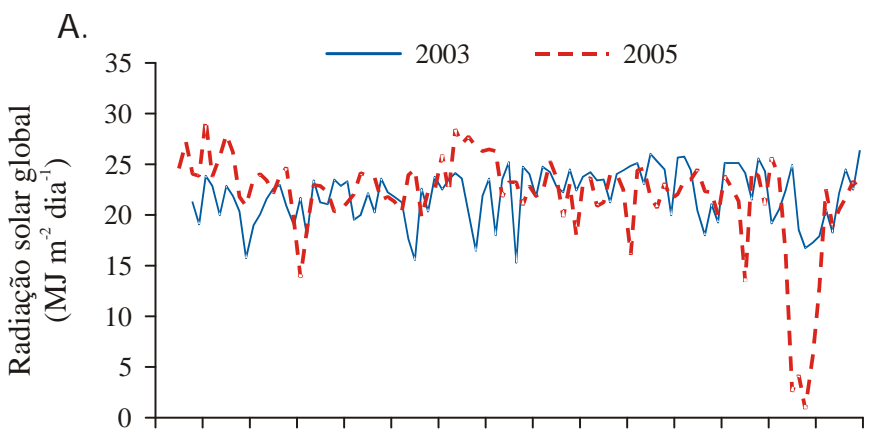

B.

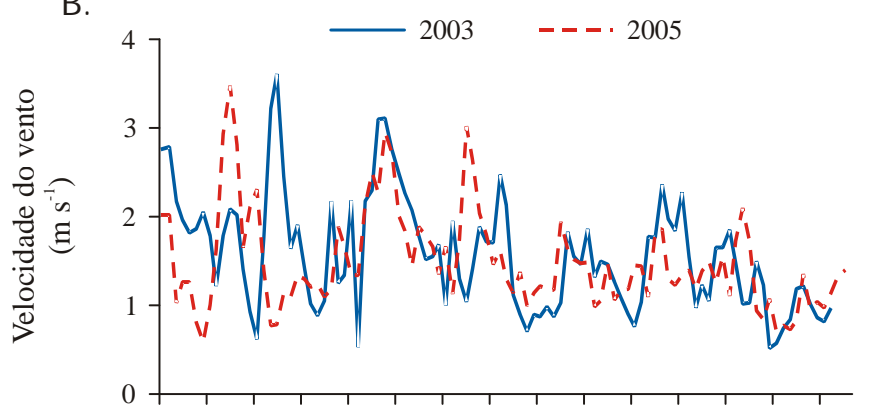

C.

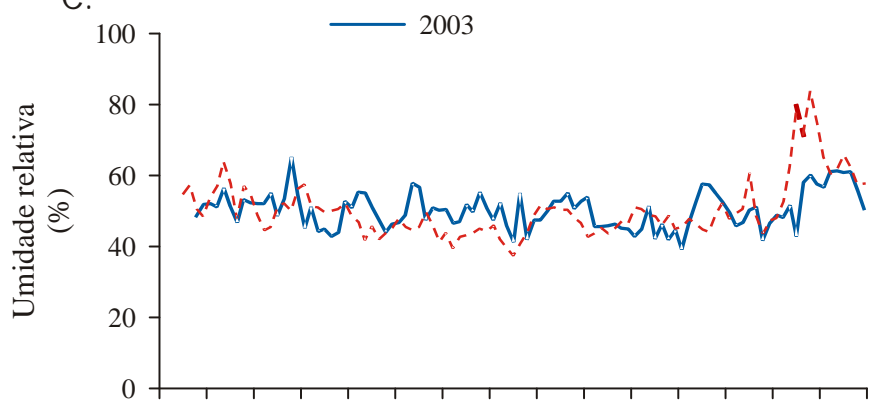

D.

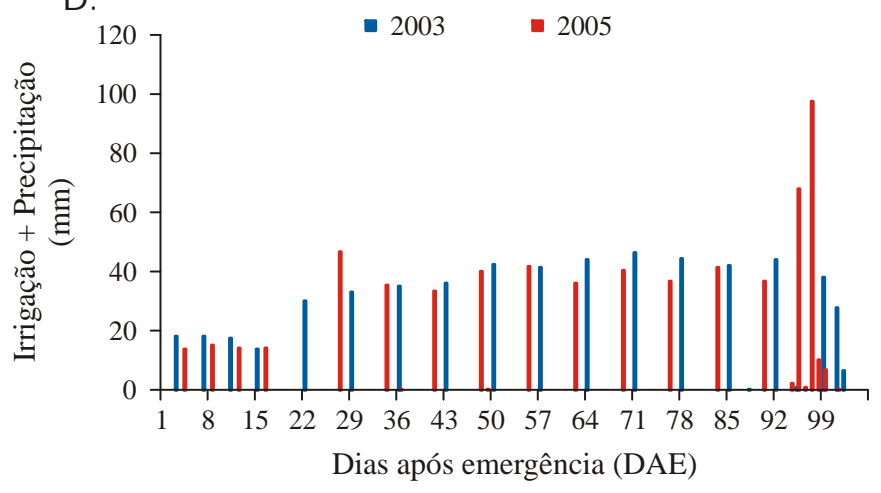

Figura 2. Variação estacional média da radiação solar global (A), velocidade do vento $(B)$, umidade relativa $(C)$ e irrigação ou precipitação $(D)$, ocorrida no período de cultivo do algodoeiro BRS-200 Marrom irrigado, em Barbalha, CE, para os anos de 2003 e 2005

cia da planta, há uma redução no consumo hídrico da cultura em decorrência da redução da área foliar provocada pela queda das folhas, conforme observado por Bezerra et al. (2005), Fidelis Filho et al. (2005) e Rodrigues (2003). Por outro lado, avaliando a altura de plantas do algodoeiro, Beltrão et al. (2000) e Rodrigues (2003), observaram que a cultura apresentou um crescimento lento no início do ciclo, seguido de um crescimento bastante rápido na fase intermediária, tendendo
Tabela 4. Valores médios da evapotranspiração (ETC) do algodoeiro, cultivar BRS-200 Marrom, por fase fenológica

\begin{tabular}{llcc}
\hline \multicolumn{1}{c}{ Fases de desenvolvimento } & $\begin{array}{c}\text { ETc médio } \\
\left(\mathbf{m m ~ d}^{\mathbf{1}} \mathbf{)}\right.\end{array}$ & $\begin{array}{c}\text { ETc do } \\
\text { período } \\
(\mathbf{m m})\end{array}$ \\
Fase I & Emergência a 10\% de cobertura do solo & 3,8 & 57,0 \\
Fase II & 10\% de cobertura do solo ao início da floração & 5,0 & 194,7 \\
Fase III Início da floração ao início da maturação & 5,9 & 183,2 \\
Fase IV & Início ao final da maturação & 5,4 & 108,4 \\
\hline & Total & - & 543,3 \\
\hline
\end{tabular}

para uma estabilização no final do ciclo.

O consumo total de água do algodoeiro, cultivar BRS-200 Marrom, estimado pela Razão de Bowen, foi $543,3 \mathrm{~mm}$, resultados semelhantes àqueles obtidos por Alves et al. (2005). Para o algodoeiro herbáceo, cultivar CNPA Precoce 1, Azevedo et al. (1993), utilizando uma bateria de evapotranspirômetros de lençol freático constante, obtiveram um consumo de $440,0 \mathrm{~mm}$. Utilizando esta mesma metodologia, Bezerra et al. (1994) encontraram um consumo hídrico total de $616,49 \mathrm{~mm}$ para a cultivar CNPA $6 \mathrm{H}$. Sob condições de cultivo protegido, Pereira et al. (1997) conseguiram consumos médios de 501,1 e $533,5 \mathrm{~mm}$ para as cultivares de algodoeiro herbáceo CNPA Precoce 1 e CNPA 7H, respectivamente.

Na Figura 3 apresenta-se, o valor médio dos dois anos de observação, para o coeficiente de cultivo $\left(\mathrm{K}_{\mathrm{c}}\right)$ diário ao longo do ciclo de desenvolvimento do algodoeiro, cultivar BRS-200 Marrom e o coeficiente de cultivo ajustado para esta cultivar. Observando-se a curva de coeficiente ajustada, verifica-se que o aumento do Kc entre as fases I e III é praticamente linear, mantendo-se aproximadamente constante ao longo da fase de maturação (Fase IV). Este comportamento se deve ao fato de que, sendo a BRS-200 Marrom uma cultivar semiperene, continua vegetando durante a fase de maturação e abertura dos capulhos. Desta forma, o coeficiente de cultivo do algodoeiro, cultivar BRS-200 Marrom irrigado, cultivado na região de Barbalha, CE, pode ser estimado pela equação de regressão: $\mathrm{Kc}=$ $-0,00006 \mathrm{DAE}^{2}+0,009 \mathrm{DAE}+0,632, \mathrm{R}^{2}=0,722$, para $\mathrm{o}$ primeiro ano de cultivo.

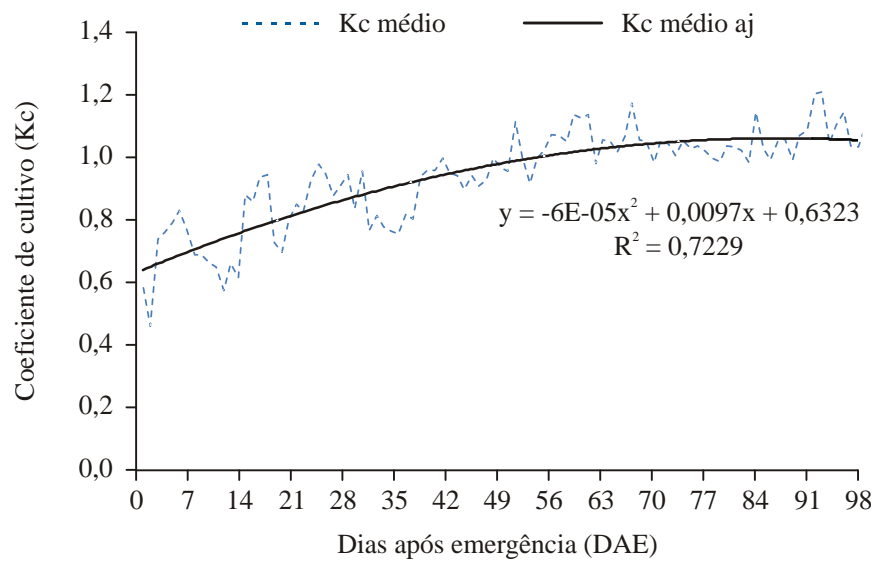

Figura 3. Coeficiente de cultivo (Kc) e coeficiente de cultivo ajustado (Kc aj) do algodoeiro BRS-200 Marrom, média dos anos 2003 e 2005 


\section{CONCLUSÕES}

1. A evapotranspiração da cultura (ETc) do algodoeiro, cultivar BRS-200 Marrom, foi de 3,8; 5,0; 5,9 e $5,4 \mathrm{~mm} \mathrm{~d}^{-1}$ para as fases I, II, III e IV, do ciclo fenológico da cultura, respectivamente.

2. O coeficiente de cultivo $(\mathrm{Kc})$ da cultura pode ser estimado pela equação $\mathrm{Kc}=-0,00006 \mathrm{DAE}^{2}+0,009 \mathrm{DAE}+0,632$.

\section{LITERATURA CITADA}

Allen, R. G.; Pruit, W. O.; Wright, J. L.; Howell, T. A.; Ventura, F.; Snyder, R.; Itenfisu, D.; Steduto, P.; Berengena, J. Yrisarry, J. B.; Smith, M.; Pereira, L. S.; Raes, D.; Perrier, A.; Alves, I.; Walter, I.; Elliott, R. A recommendation on standardized surface resistance for hourly calculation of reference ETo by the FAO56 Penman-Monteith method. Agricultural Water Management, v.81, p.1-22, 2006.

Alves, J. C. M.; Pereira, J. R.; Bezerra, J. R. C.; Lima, S. V. de; Souza, R. N. de; Mascena, A. M.; Pinho Filho, F. de A. L. de; Alencar, S. de B. Partição de assimilados em algodoeiro semiperene BRS Marrom sob diferentes lâminas de irrigação e doses de regulador de crescimento. In: Congresso Brasileiro de Algodão, 5., 2005, Salvador. Anais...Campina Grande: Embrapa Algodão, 2005. CD-Rom

Amorim Neto, M. da S. Termometria a infravermelho associada ao balanço de energia na determinação do índice de estresse hídrico da cultura do feijoeiro (Phaseolus vulgaris L.) irrigado. Viçosa: UFV, 1995. 89p. Tese Doutorado

Azevedo, P. V. de; Rao, T. V. R.; Amorim Neto, M. da S.; Bezerra, J. R. C.; Espínola Sobrinho, J.; Maciel, G. F. Necessidades hídricas da cultura do algodoeiro. Pesquisa Agropecuária Brasileira, v.28, n.7, p.863-870, 1993.

Azevedo, P. V. de; Silva, B. B. da; Silva, V. de P. R. Water requirements of irrigated mango orchards in northeast Brazil. Agricultural Water Management, v.58, p.241-254, 2003.

Beltrão, N. E. de M.; Fidelis Filho, J.; Souza, J. G. de. Produtividade, qualidade de fibra e análise de crescimento com estimativa de respiração, do algodão perene $7 \mathrm{MH}$, nas condições ecofisiológicas do Seridó paraibano, comparado à CNPA 5M. Revista Brasileira de Oleaginosas e Fibrosas, v.4, n.1, p.13-21, 2000.

Beltrão, N. E. de M.; Lima, R. de L. S.; Leão, A. B.; Albuquerque, W.; G. de Algodão brasileiro em relação ao mundo: situação e perspectivas. In: Beltrão, N. E. de M.; Azevedo, D. M. P. de (ed.) $\mathrm{O}$ agronegócio do algodão no Brasil. Brasília: Embrapa Informação Tecnológica, 2008. p.19-30.

Bezerra, J. R. C.; Barreto, A. N.; Silva, B. B. da; Espínola Sobrinho, J.; Rao, T. V. R.; Luz, M. J. da S. e; Medeiros, J. D. de; Souza, C. B. de; Silva, M. B. da. Consumo hídrico do algodoeiro herbáceo. In: Embrapa CNPA. Campina Grande: Embrapa CNPA, 1994. p.151-154. Relatório Técnico Anual, 1992-1993.

Bezerra, J. R. C.; Luz, M. J. da S. e; Barreto, A. N.; Amorim Neto, M. da S.; Silva, L. C. Irrigação do algodoeiro. In: Beltrão, N. E. de M.; Azevedo, D. M. P. de (Org.) O agronegócio do algodão no Brasil. Brasília: Embrapa Informação Tecnologica, 2008. p. 875-949.
Bezerra, J. R. C.; Luz, M. J. da S. e; Pereira, J. R.; Santana, J. C. F. de; Dias, J. M.; Santos, J. W. dos; Santos, T. da S. Rendimento e qualidade de fibra do algodoeiro herbáceo em diferentes épocas de interrupção da irrigação. Revista Brasileira de Oleaginosas e Fibrosas, v.7, n.2/3, p.719-726, 2003.

Bezerra, L. J. D.; Lima, V. L. A. de; Andrade, A. R. S. de; Alves, V. W.; Azevedo, C. A. V. de; Guerra, H. O. C. Análise de crescimento do algodão colorido sob os efeitos da aplicação de água residuária e biossólidos. Revista Brasileira de Engenharia Agrícola e Ambiental, v.9, Suplemento, p.333-338, 2005.

Blanc, E.; Quirion, P.; Strobl, E. The climatic determinants of cotton yields: evidence from a plot in West Africa. Agricultural and Forest Meteorology, v.148, p.1093-1100, 2008.

Borges, C. J. R.; Azevedo, P. V. de; Silva, V. de P. R. da; Campos, J. H. B. da C.; Moura, M. S. B. de; Soares, J. M.; Silva, B. B. da. Influência no calor armazenado no sistema solo-planta no balanço de energia em pomar de mangueiras. Revista Brasileira de Engenharia Agrícola e Ambiental, v.12, n.4, p.393-399, 2008.

Brasil. Ministério da Agricultura e Reforma Agrária. Normais climatológicas: (1961-1990). Brasília: Departamento Nacional de Meteorologia. 1992. 84p.

Cardoso, G. B.; Viana, T. V. de A.; Azevedo, B. M. de; Sousa, V. F. de; Souza, F. de Determinação da ET de referência pela razão de Bowen com psicrômetros instalados a diferentes alturas. Revista Ciência Agronômica, v.36, n.1, p.16-23, 2005.

Doorenbos, J.; Pruitt, W. O. Las necesidades de agua de los cultivos. Roma: FAO, 1979. 194p. Riego y Drenaje, 24

Farahani, H. J.; Oweis, T. Y.; Izzi, G. Crop coefficient for drip-irrigated cotton in a Mediterranean environment. Irrigation Science. v.26, p.375-383, 2008.

Fidelis Filho, J.; Nóbrega, J. Q.; Sousa, J. T. de; Dantas, J. P. Comparação dos efeitos de água residuária e de poço no crescimento e desenvolvimento do algodoeiro. Revista Brasileira de Engenharia Agrícola e Ambiental, v.9, Suplemento, p.328-333, 2005.

Heilman, J. L.; Brittin, C. L.; Neale, C. M. U. Fetch requeriments for Bowen ratio measurements of latent and sensible heat fluxes. Agricultural Water Management, v.44, n.3-4, p.261-273, 1989.

Gavilán, P.; Berengena, J. Accuracy of the Bowen ration-energy balance method for measuring latent heat flux in a semiarid advective environment. Irrigation Science, v.25, p.127-140, 2007.

Jalota, S. K.; Sood, A.; Chahal, G. B. S.; Choudhury, B. U. Crop water productivity of cotton (Gossypium hirsutum L.) - Wheat (Triticum, aestivum L.) system as influenced by deficit irrigation, soil texture and precipitation. Agricultural Water Management, v.84, p.137-146, 2006.

Lopes, P. M. O.; Silva, B. B. da; Azevedo, P. V. de; Silva, V. de P. R. da; Teixeira, A. H. de C; Soares, J. M.; Espínola Sobrinho, J. Balanço de energia num pomar de mangueira irrigado. Revista Brasileira de Agrometeorologia, v.9, n.1, p.1-8, 2001.

Luz, M. J. da S. e; Bezerra, J. R. C.; Santana, J. C. F. de; Dias, J. M. Efeito da deficiência hídrica sobre o rendimento e a qualidade de fibra do algodoeiro. Revista Brasileira de Oleaginosas e Fibrosas, v.1, n.1, p.125-133, 1997. 
Pereira, M. do N. B.; Azevedo, N. C.; Fernandes, P. D.; Amorim Neto, M. da S.; Crescimento e desenvolvimento de duas cultivares de algodoeiro herbáceo em baixos níveis de umidade no solo, em casa de vegetação. Revista Brasileira de Engenharia Agrícola e Ambiental, v.1, p.1-7, 1997.

Perez, P. J.; Castellvi, F.; Martinez-Cob, A. A simple model for estimating the Bowen ratio from climatic factors for determining latent and sensible heat flux. Agricultural Forest Meteorology, v.148, p.25-37, 2008.

Prueger, J. H.; Hatfield, J. L; Aase, J. K.; Pikul Jr., J. L. Bowenratio comparisons with lysimeter evapotranspiration. Agronomy Journal, v.89, n.5, p.730-736, 1997.

Radin, J. W.; Reaves, L. L.; Mauney, J. R.; French, O. F. Yield enhancement in cotton by frequent irrigations during fruiting. Agronomy Journal, v.84 p.551-557, 1992.

Reis, L. S.; Souza, J. L. de; Azevedo, C. A. V. de. Evapotranspiração e coeficiente de cultivo do tomate caqui cultivado em ambiente protegido. Revista Brasileira de Engenharia Agrícola e Ambiental, v.13, n.3, p.289-296, 2009.

Rodrigues, M. F. G. Necessidades hídricas, crescimento e desenvolvimento do algodoeiro herbáceo, cultivar BRS 201. Campina Grande: 2003. 132p. Tese Doutorado

Rosenberg, N. J.; Blad, B. L. Verma, S. B. Microclimate: The biological environment. New York: John Wiley, 1983. 495p.

Silberstein, R. P.; Sivapalan, M.; Viney, N. R; Held, A.; Jatton, T. $\mathrm{J}$. Modelling the energy balance of a natural jarrah (Eucalyptus marginata) forest. Agricultural and Forest Meteorology, v.115, n.3-4, p.201-230, 2003.

Silva, B. B. da; Rao, T. V. R. The CWSI variations of a cotton crop in a semi-arid region of Northeast Brazil. Journal of Arid Environments, v.62, p.649-659, 2005.

Silva, V. de P. R.; Azevedo, P. V. de; Silva, B. B. da. Surface energy fluxes and evapotranspiration of a mango orchard grown in Semiarid environment. Agonomy Journal, v.99, n.6, p.1391-1396, 2007.
Souza, C. B. de. Necessidades hídricas, crescimento e desenvolvimento do abacaxizeiro nos tabuleiros de Santa Rita - PB. Campina Grande: UFPB, 2003. 124p. Tese Doutorado

Souza, C. C. de; Oliveira, F. A. de; Silva, I. de F. da; Amorim Neto, M. da S. Avaliação de métodos de determinação de água disponível em solo cultivado com algodão. Pesquisa Agropecuária Brasileira, v.37, n.3, p.337-341, 2002.

Stannard, D. I.; Rosenberry, D. O.; Winter, T. C.; Parkhurst, R. S. Estimates of fetch-induced errors in Bowen-ratio energy-budget measurements of evapotranspiration from a prairie wetland, cottonwood lake area, North Dakota, USA. Wetlands, v.24, n.3, p.498-513, 2004.

Teixeira, A. H. C.; Bastiaanssen, W. G. B.; Bassoi, L. H. Crop water parameter of irrigated wine and grapes to support water productivity analysisin the São Francisco river basin, Brazil. Agricultural Water Management, v.94, n.1-3, p.31-42, 2007.

Thornthwaite, C. W.; Mather, J. R. The water balance. Centerton: Drexel Institute of Technology, 1955. 104p. Publications in Climatology, v.8, n.1

Verma, S. B.; Rosemberg, N. J.; Blad, B. L. Turbulent exchange coefficients for sensible eat and water vapor under advective conditions. Journal of Applied Meteorology, v.17, p.330-338, 1978.

Vourlitis, G. L.; Priante Filho, N.; Hayashi, M. M. S.; Nogueira, J. de S.; Caseiro, F. T.; Campelo Jr., J. H. Seasonal variations in the evapotranspiration of a transitional tropical forest of Mato Grosso, Brazil. Water Resources Research, v.38, n.6, p.30/1-30/11, 2002.

Zhang, B.; Kang, S.; Li, F.; Zhang, L. Comparison of three evapotranspiration models to Bowen ratio-energy balance method for a vineyard in an arid desert region of northwest China. Agricultural and Forest Meteorology, v.148, n.10, p.1629-1640, 2008. 